\title{
Predicting Location Using Mobile Phone Calls
}

\author{
Daqiang Zhang \\ Nanjing Normal University \\ Nanjing, 210046, China \\ dqzhang@njnu.edu.cn
}

\author{
Athanasios V. Vasilakos \\ National Technical University \\ Athens, 15780, Greece \\ vasilako@ ath.forthnet.gr
}

\author{
Haoyi Xiong \\ Institute Mines Telecom \\ Paris, 91011, France \\ hyxiong@ieee.org
}

\begin{abstract}
Location prediction using mobile phone traces has attracted increasing attention. Owing to the irregular user mobility patterns, it still remains challenging to predict user location. Our empirical study in this paper shows that the call patterns are strongly correlated with co-locate patterns (i.e., visiting the same cell tower at the same period), and the call patterns mainly affect user short-time mobility. On top of these findings, we propose NextMe - a novel scheme to enhance the location prediction accuracy by leveraging the social interplay revealed in the cellular calls. To identify when the social interplay will affect user mobility, we introduce the concepts of the Critical Call Pattern (CCP), and the Critical Call (CC). We validate NextMe with the MIT Reality Mining dataset, involving 350,000-hour activity logs of 106 persons, and 112,508 cellular calls. Experimental results show that the social interplay significantly improves the accuracy.
\end{abstract}

\section{Categories and Subject Descriptors}

J.4 [Computer Application]: Social and Behaviorial Sciences; D.4.8 [Performance]: Prediction

\section{General Terms}

Algorithms, Experimentation, Measurement

\section{Keywords}

Social Networks, Mobile Phone Calls, Social Interplay

\section{INTRODUCTION}

Mobile devices have become a ubiquitous part of user daily lives such that they capture a great many of mobile traces including user cell IDs, cellular calls, short messages and activity logs. These traces provide insights into user behavior, location and proximity and thus open a new avenue for location prediction.

A variety of location prediction schemes have been proposed in recent years. Some schemes, e.g., PMM [2], foretell user location based on an intuition that the majority of human mobility is periodic among a small set of location. Some schemes make location prediction by the social relationships, e.g., HCMM [1]. However, existing schemes do

Copyright is held by the author/owner(s). SIGCOMM'12, August 13-17, 2012, Helsinki, Finland. ACM 978-1-4503-1419-0/12/08.

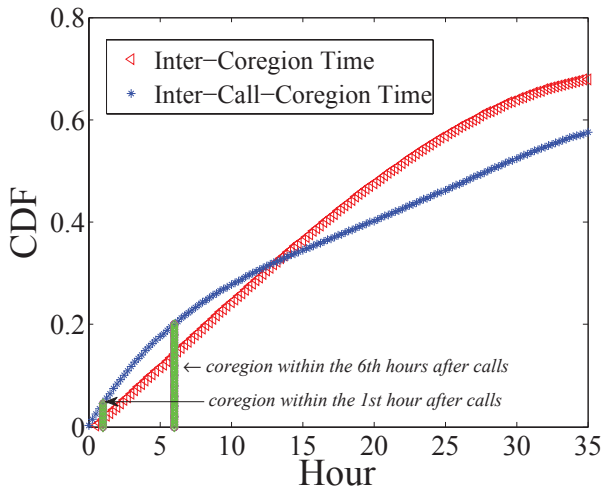

Figure 1: The CDF diagrams

not clearly tell when the social relationships affect user mobility. Moreover, they rely on other data source than mobile phone traces to identify user relationship, e.g., extracting friendships from Facebook connections.

To this end, we investigate the location prediction using the MIT Reality Mining dataset that is one of most popular mobile traces, involving 1,000,000 GSM traces and 112,508 mobile calls ${ }^{1}$. We find that the cellular call pattern between two users is highly correlated with their co-location (i.e., simultaneously co-locate at the same cell tower), and it mainly influences user mobility in a short period. User pairs who follow a certain call pattern will co-locate soon after calls with a great probability. Consequently, we introduce the social interplay concept to reveal the social relationship embedded in mobile calls, and propose a predictor named NextMe on top of it. To invoke the NextMe predictor, we further introduce the concepts of the Critical Call Pattern (CCP) and the Critical Call (CC).

\section{PRELIMINARY FINDINGS}

We report our preliminary findings by two questions: 1) does the social interplay exist in mobile calls, and 2) when will the social interplay affect the user mobility. We extract two kinds of events from the MIT Reality Mining dataset - the mobile call that denotes a direct call from one use to another, and the co-locate event (i.e., coregion event) that stands for two users visiting the same cell tower at the same period of time.

In order to answer the first question, we present two con-

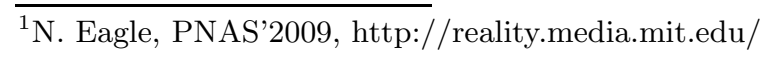


cepts - the inter-coregion time and the inter-call-coregion time. The former concept is the interval between two consecutive co-locate events, whereas the later one denotes the interval between the cellular call time and the next co-locate time. Their CDFs are illustrated as Fig. 1 . When the prediction time varies from 1 to 12 , the CDF of inter-call-coregion time is higher than that of the inter-coregion time. This figure has two important implications. Firstly, the social interplay as one kind of social relationship does exist in mobile calls. Secondly, it has significant impact on user short-time mobility. This indicates that the social interplay will drive user to move for co-location within next few hours after critical mobile calls.

Regarding the second question, we plan to investigate what kinds of cellular call patterns would coincide with the co-locate event. We find a certain Cellular Call Pattern (CCP), which makes a call pair have an immediate co-locate event with a very high likelihood. Let $n$ be the number of cellular calls occurred during the last co-locate event and the current call time and $\lambda$ be the interval between the last two mobile calls. Apparently, all $n$ follows the Gaussian distribution. Thus, we leverage the Gaussian Parameter Estimation to get its confidence interval $\left[n_{1}, n_{2}\right]$ with the confidence level as 0.95 . For the same reason, we obtain the confidence interval $\left[\lambda_{1}, \lambda_{2}\right]$ for the parameter $\lambda$.

Definition 1. A Critical Call Pattern (CCP) between two successive co-locate events is a call sequence that satisfies: 1) $n_{1} \preceq n \preceq n_{2}$, and 2) $\lambda_{1} \preceq \lambda \preceq \lambda_{2}$.

Definition 2. Given a CCP, the Critical Call (CC) refers to the last cellular call.

With the CCP and the CC, we can easily detect the moment social interplay might affect user mobility. Note that there may be other call patterns such that the call pair will co-locate soon after calls.

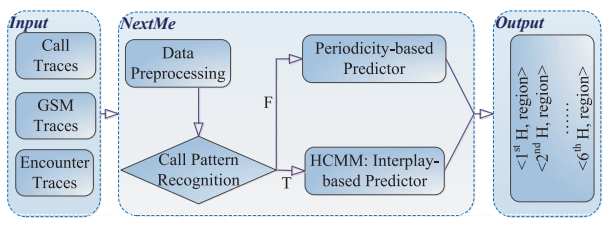

Figure 2: The designed architecture

\section{NEXTME SCHEME}

In this section, we propose NextMe scheme that leverages both user behavior regularity and social interplay to predict user location at cell tower level.

Fig. 2 illustrates the system architecture of the proposed scheme, comprising data preprocessing, call pattern recognition, the periodicity-based predictor PMM [2] and the social interplay-based predictor HCMM [1]. The call pattern recognition module identifies when the social interplay-based predictor works. By Definition 1, it will recognize the CCP. By Definition 2, it easily identifies the $\mathrm{CC}$ that triggers the social interplay-based predictor. Once a CCP is detected, NextMe will predict user location using the social interplay, otherwise using the behavior regularity.

Specifically, NextMe collects the inter-call-coregion time of a given call pair from the Reality dataset. Then, it obtains

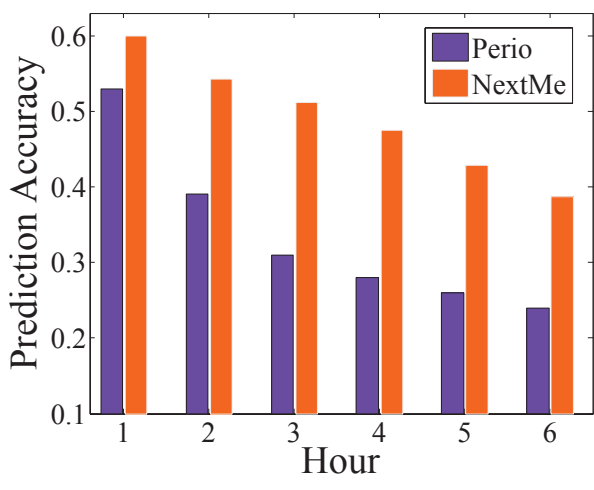

Figure 3: The overall performance of the $P M M$ and NextMe

the Gaussian distribution of the inter-call-coregion time by parameter estimation. It computes the PDF of the intercall-coregion time. According to the time that CCs occurred and the PDF, NextMe infers the moment that the call pair will co-locate.

Given a call pair $\left(u_{x}, u_{y}\right)$, let $\tau$ be the prediction duration (i.e., its value as one of 1 to 6 hours), $T_{i}$ be the $i^{\text {th }}$ call in the $n^{t h}$ successive calls in a CCCP, $K^{u_{x}, u_{y}}(\tau)$ is a likelihood function for the $\tau^{t h}$ prediction hour, and $G_{u_{x}, u_{y}}$ be the Gaussian function. NextMe obtains the coregion probability $P_{u_{x}, u_{y}}(\tau)$ for user pair $u_{x}$ and $u_{y}$ at the $\tau^{\text {th }}$ hour as Formula 1.

$$
\begin{aligned}
& \underset{\tau}{\arg \max } P_{u_{x}, u_{y}}(\tau) \text { and } \tau \in\{1,2, \ldots, 6\} \\
& P_{u_{x}, u_{y}}(\tau)=P_{u_{x}, u_{y}}^{1-6} \bullet \frac{K_{u_{x}, u_{y}}(\tau)}{\sum_{\tau \in\{1,2, \ldots, 6\}} K_{u_{x}, u_{y}}(\tau)}
\end{aligned}
$$

, where $K_{u_{x}, u_{y}}(\tau)$ is computed as Formula 2:

$$
K_{u_{x}, u_{y}}(\tau)=\prod_{1 \leq i \leq n} G_{u_{x}, u_{y}}\left(T_{n}-T_{i}+\tau\right), \tau \in(1,6)
$$

We select the PMM as the baseline, and the aggregated accuracy as the metric that is computed as the average prediction accuracy of all participants. Fig. 3 illustrates the overall performance of NextMe, where the $\mathrm{x}$-axis is the prediction hour after calls. It shows that NextMe achieves higher accuracy than $P M M$ in all predictions. This implies that the social interplay dramatically affects user short-time mobility and contributes $13 \%$ on average to accuracy.

\section{CONCLUSIONS}

In this paper, we have uncovered the social interplay revealed in mobile calls. We further propose NextMe scheme on top of user behavior regularity and social interplay. Initial results show that the social interplay has a significant affect on user short-time mobility.

\section{REFERENCES}

[1] C. Boldrini and A. Pass. Modelling spatial and temporal properties of human mobility driven by users' social relationships. Comp. Comm., 33:1056-1074, 2010.

[2] E. Cho et al. Friendship and mobility: user movement in location-based social networks. In Proc. of the SIGKDD, pages 1082-1090, 2011. 